\title{
JPIAspectZ: A Formal Requirement Specification Language for Joint Point Interface AOP Applications
}

\author{
Cristian Vidal-Silva \\ Faculty of Economics and Administration, \\ Catholic University of the North, \\ Antofagasta, Chile \\ cristian.vidal@ucn.cl \\ Trung Pham \\ Faculty of Economics and Business, \\ University of Talca, \\ Talca, Chile \\ tpham@utalca.cl \\ Luis Alberto Urzua \\ Faculty of Health, \\ Santo Tomas University, \\ Chile Talca, Chile \\ lurzua@santotomas.cl
}

\author{
Erika Madariaga \\ Faculty of Engineering, \\ Bernardo O'Higgins University, \\ Santiago, Chile \\ erika.madariaga@ubo.cl
}

Franklin Johnson

Computing and Information Department, University of Playa Ancha,

Valparaiso, Chile

franklin.johnson@upla.cl

\author{
Luis Carter \\ Ingenieria Civil Industrial, \\ Facultad de Ingenieria, \\ Universidad Autonoma de Chile, Chile \\ luis.carter@uautonoma.cl
}

\begin{abstract}
Aspect-oriented software development (AOSD) solves a few issues of the object-oriented software development (OOSD) approach and adds a few more concerning modules and their relationships. Join point interface (JPI) is an AOSD methodology that by the definition of the interface between advised artifacts and aspects solves associated AOSD issues to get software with a high modularity level. Looking for a JPI software development approach, this article proposes and exemplifies the use of JPIAspect $Z$, an extension of the formal aspect-oriented language AspectZ for the software JPI requirement specifications. Mainly, JPIAspectZ looks for a concept and model consistency in a JPI software development process. Since the main JPI characteristics are the joining point interfaces definitions, i. e. explicit associations definition between aspects and advised modules, thus, by JPI, classes are no longer oblivious of possible interaction with aspects, and aspects, for their action effectiveness, do not depend anymore on signatures of advisable module components. JPIAspect $Z$ fully supports these JPI principles. As JPI application examples, this article shows the formal requirements specification for classic aspect-oriented and JPI examples, along with describing the advantages and disadvantages of this language.
\end{abstract}

Keywords-JPIAspectZ; JPI; AspectZ; aspects; join point interface

\section{INTRODUCTION}

AOSD permits modularizing crosscutting concerns in OOSD stages [1]. Because AOSD was born at the objectoriented (OO) programming stage, to reach a complete transparency of concepts and design in the AOSD process seems a complex task. Looking for a transparency in the AOSD process, different proposals of modeling language extensions already exist to support AOSD such as aspectoriented UML use case diagrams [2] and aspect-oriented UML class diagram [3]. Authors in [4] present a survey of aspectoriented UML language proposals. Nevertheless, only a few articles about formal aspect-oriented languages for requirement specification exist so far. Authors in $[5,6]$ describe and apply AspectZ, authors in $[7,8]$ describe OOAspectZ, and authors in [9] illustrate the use of an aspect-oriented alloy version. Authors in [10] report that a double-dependency between base modules and aspects exists in traditional AOSD solutions. To solve this issue, the works of [10-12] propose and apply join point interface (JPI) instances between classes and aspects. Thus, with the purpose of obtaining JPI solutions and getting transparency of concepts in stages of the AOSD-JPI process, this article proposes and applies JPIAspectZ, an extension of OOAspectZ [7, 8], for requirements specification of JPI software applications.

\section{ASPECT ORIENTED PROGRAMMING AND JPI}

Authors in [1] proposed aspect-oriented programming (AOP) to modularize crosscutting concerns as aspects in OOP. Aspects advise classes like events, i.e. aspects introduce behavior and structural elements such as methods and attributes into classes. Nevertheless, as authors in [10] indicate, AOP presents implicit dependencies between advised classes and 
aspects. First, aspects define pointcut rules (PCs) for advisable class behavior, and, as a result, instances of those classes are entirely oblivious of possible changes in their components, methods, and attributes. Second, aspects can be ineffective or spurious for signature changes on advised methods of target classes. As authors in $[10,11]$ mention, the last issue is known as the fragile pointcut problem. Authors in [10] indicate that traditional AOP like Aspect-J solutions compromise the independent development of base code and aspect modules since developers of base code, and aspects must obtain a global knowledge about all program components and their associations, i.e. they must know all the details about aspects, classes, and their relations. To isolate crosscutting concerns and get modular AOP programs without the mentioned implicit dependencies, authors in [10] describe the JPI programming methodology. JPI introduces the idea of join point interface on classic AOP. Like classic AOP [10, 11], for JPI applications, aspects represent crosscutting functionalities, but without PCs. Aspects in JPI only present their implementation of join point interfaces. Besides, in JPI, non-oblivious advised classes exhibit explicit join point interfaces, that is, classes know about potential changes on their methods. Figures 1 and 2 [10] illustrate dependencies between aspects and classes in classic AOP and JPI applications, respectively.

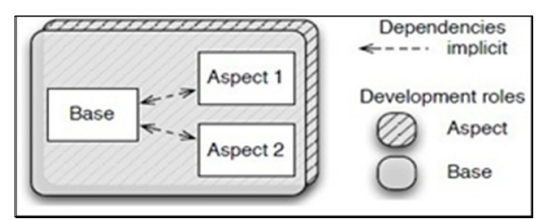

Fig. 1. Base and aspects modules association in classic AOP.

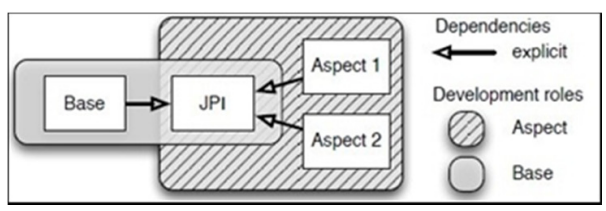

Fig. 2. Base and aspect modules association in JPI.

\section{JPIASPECTZ PRINCIPLES}

$Z$ [13] and Object-Z [14] are formal languages for software requirement specification. Specifically, $Z$ is the classic formal specification language without the direct support of objectoriented abstractions like classes and inheritance, and Object- $Z$ is an extension of $Z$ to support OOSD principles. Likewise, AspectZ [5, 6] and OOAspectZ [7, 8] represent $Z$ extensions for requirements specification of AOP applications and their integration with $Z$ and Object-Z, respectively. Considering JPI ideas, this article describes JPIAspectZ, an OOAspectZ extension to model JPI applications and its integration with Object-Z. The main elements of a JPIAspectZ formal specification are:

Base Modules: Unlike AspectZ and OOAspectZ which present oblivious base modules, JPIAspectZ base modules are specified as Object- $Z$ class modules which include an exhibit rule concerning advisable operations of advised class instances. Figure 5 shows the structure of a JPIAspectZ class schema, JPI schema, and Aspect schema. Since the declaration part of an
Object-Z operation schema permits defining operation parameters, when looking for a transparency of concepts and design for JPI applications, an exhibit rule is definable in two sections: first, exhibits JPI for the join point interface instances which the class exhibits, and second, a set of conditions for the join point event. So far, JPIAspectZ considers basic AOP and JPI conditions for dynamic and static crosscuts, i.e. call operation, execution operation, logic connectors \&\&, \|, !; args (arguments list) to identify catchable method arguments, this(object) to determine the object on which the advisable method operates, and target(object) to identify the object owner of the advisable method.

Join Point Interface: In JPIAspectZ, operation schemas starting with the JPI initials represent join point interfaces (JPI schemas) for a system specification. For example, Figure 6 shows JPIUpdateX and JPIUpdateY. Furthermore, JPI schemas only present a declaration section to indicate their list of parameters.

Aspects: JPIAspectZ Aspects-schemas are like ObjectZ class diagrams labeled with the phrase aspect. Aspect-schemas include state schemas to define attributes and invariants and operation schemas for the schema advice operations. As a distinction regarding class schemas, aspect-schemas can indicate the occurrence time for operations (before, after, and around) to specify the kind of advice. Semantically, aspectschemas advise operation schemas, usually for inserting new methods in the advised classes, for adding behavior at the beginning, around, and end on advised operations schemas. From advised method schemas and associated aspect schemas, JPIAspectZ permits obtaining woven schemas. It is relevant to highlight the modular evolution from AspectZ, OOAspectZ, and JPIAspectZ schemas as Figure 3 [5], Figure 4 [7, 8], and Figure 5 respectively present. Note that for the first two, base schema, Z operation schema and ObjectZ class schema, AspectZ aspects operate over oblivious advised elements. Nevertheless, for the JPI philosophy, in JPIAspectZ, the aspects and classes know about interfaces to implement and exhibit, respectively.

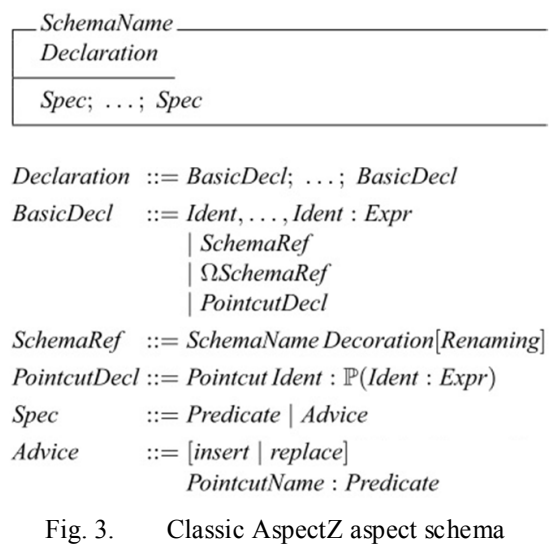

As Figure 5 describes, JPIAspectZ considers advisable classes which exhibit advisable operations in their state schema (Figure 5(a)), Join Point Interface (JPI) schemas as a link between advisable classes and aspects adviser, and aspects who 
will advise those classes in the ocurrence of join points in objects of the class (Figure 5(b)).

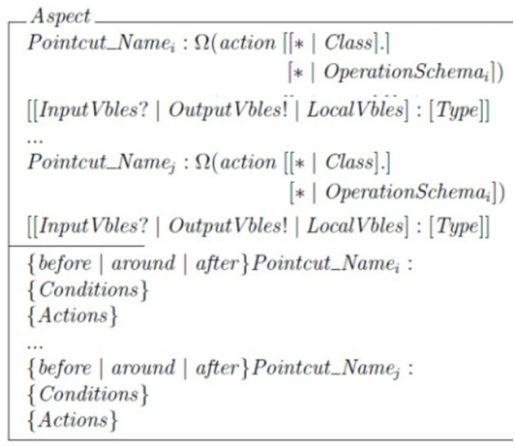

Fig. 4. OOAspectZ aspect schema.

(a)

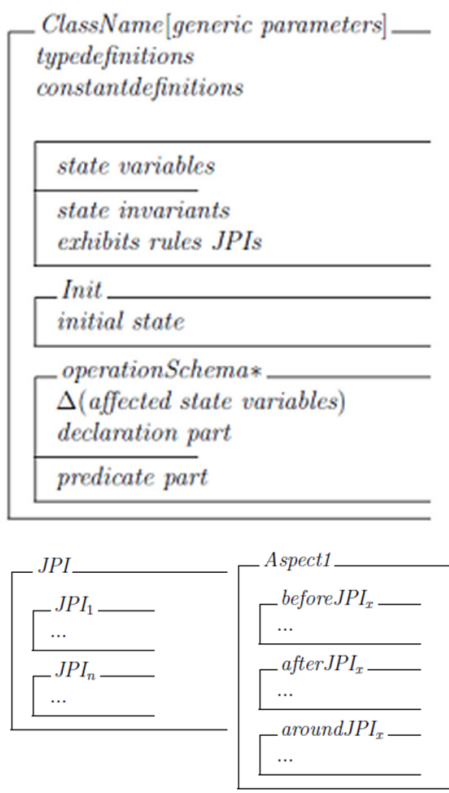

Fig. 5. Base and aspect modules association in JPIAspectZ: (a) Advised class schema, (b) JPI and aspect-schemas

\section{APPLICATION EXAMPLES}

The Painting System [7] is a classic AO example that presents classes Point and Line which are Shapes, and each Line instance is composed of a few Point instances. The main idea is to illustrate the updating screen process as an external behavior. An illustration of the UML class diagram can be seen in [7], which presents an interface Shape to enclose Point and Line classes, and each Line instance is composed of two Point instances, P1 and P2. Note that for classic AO, both classes obliviously wait for advices from the aspect UpdateSignaling concerning the pointcut rules definition outside the classes. A JPI UML class diagram that includes the main JPI elements for the Painting System, that is, non-oblivious classes which exhibit JPI instances and aspect that implements those interfaces can be seen in [15]. Clearly, for exhibits and implements rules, classes are not more oblivious, and aspect does not directly refer to classes: classes exhibit JPI and aspects implement those interfaces. We recommend reviewing
[15] for more details about JPI. As a JPI example, [10, 12] show a Shopping session 'running example' of an e-commerce system (ShoppingSession system). This example presents a join point interface checkingOut, a class ShoppingSession that exhibits checkingOut and an aspect Discount that implements checkingOut for around kind of advice.

(a)
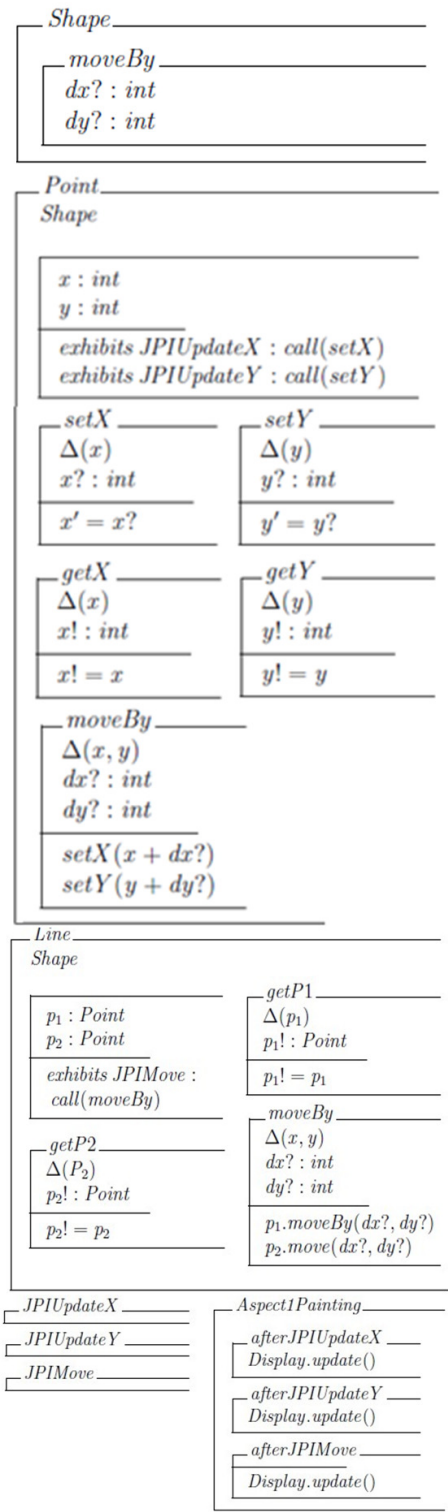

Fig. 6. Painting system JPIAspectZ formal specification (a) advised class schema, (b) advisable class Point, (c) advised class Line, (d) JPI instances and Aspect-Schema

\section{EXPERIMENTS AND RESULTS}

Figure 6 presents the JPIAspectZ specification for the Painting system: a Shape interface, Point and Line classes, JPI instances JPIUpdateX, JPIUpdateY, and JPIMove, and aspect Aspect1Painting. Point and Line classes exhibit JPI instances, class Point exhibit JPIUpdateX and JPIUpdateY, and class Line exhibit JPIMove, whereas AspectlPainting implements these JPI instances. Figure 6 presents the model's consistency. 


\section{CONCLUSIONS}

After reviewing JPIAspectZ examples for the Painting and Shop-pingSession systems, we can argue a clear consistency exists between JPI models and JPI code. Furthermore, JPIAspectZ includes JPI practical details such as exhibits and association among JPI components, that i.e., aspects, JPI, and classes to represent complete JPI applications using called external advises is possible. Thus, JPIAspectZ permits formal models of JPI applications without closure join points [16].

This article presented JPIAspectZ that permits specifying formal requirements for JPI applications, i.e. nondependent classes and aspects according to the JPI central principle. Besides, this extended JPI abstraction demonstrates that consistency and transparency of models and concepts for a JPI software development process is attainable, specifically, a consistency between requirements and structural models, and requirements and code. To achieve real consistency between structural models and JPI solutions code modules seems direct. A formal proof of this consistency represents a future work scope for the authors. Furthermore, considering future work, authors want to continue proposing extensions on JPIAspectZ to model closure join points as well as more advanced dynamic crosscuts [16]. Besides, we propose the developing of a JPIAspectZ specification validation tool for automatic validation of JPIAspectZ specifications.

\section{REFERENCES}

[1] G. Kiczales, "Aspect-oriented programming”, ACM Computing Surveys, Vol. 28, No. 4, 1996

[2] I. Jacobson, P. W. Ng, Aspect-Oriented Software Development with Use Cases, Addison Wesley Professional, 2004

[3] F. Wedyan, S. Ghosh, L. R. Vijayasarathy, "An approach and tool for measurement of state variable based data-flow test coverage for aspectoriented programs", Information and Software Technology, Vol. 59, pp. 233-254, 2015

[4] M. Wimmer, A. Schauerhuber, G. Kappel, W. Retschitzegger, W. Schwinger, E. Kapsammer, "A survey on UML based aspect-oriented design modeling”, ACM Computing Surveys, Vol. 43, No. 4, pp. 28:128:59, 2011

[5] Y. Huiqun, L. Dongmei, Y. Li, H. Xudong, "Formal Aspect-Oriented Modeling and Analysis by Aspect-Z", 17th International Conference on Software Engineering and Knowledge Engineering, Taipei, Taiwan, July 14-16, 2005

[6] C. V. Silva, R. Saens, C. Del Rio, R. Villarroel, "Aspect-oriented modeling: Applying aspect-oriented UML use cases and extending aspect-z", Computing and Informatics, Vol. 32, No. 3, pp. 573-593, 2013

[7] C. V. Silva, R. Saens, C. Del Rio, R. Villarroel, "OOAspectZ y diagramas de clase orientados a los aspectos para la modelacion orientada a aspectos (MSOA)", Ingenieria e Investigacion, Vol. 33, No. 3, pp. 66-71, 2013 (in Spanish)

[8] C. V. Silva, R. Villarroel, R. S. Simon, R. Saens, T. Tigero, C. Del Rio, "Aspect-Oriented Formal Modeling: (AspectZ + Object-Z) = OOAspectZ", Computing and Informatics, Vol. 34, No. 5, pp. 9961016,2015

[9] F. Mostefaoui, J. Vachon, "Verification of Aspect-UML Models Using Alloy", 10th International Workshop on Aspect-Oriented Modeling, Vancouver, Canada, March 12-12, 2007

[10] E. Bodden, E. Tanter, M. Inostroza, "Join point interfaces for safe and flexible decoupling of aspects", ACM Transactions on Software Engineering and Methodology, Vol. 23, No. 1, pp. A:1-A:42, 2014

[11] E. Bodden, "Closure Join Points: Block Join Points without Surprises", Tenth International Conference on Aspect-Oriented Software Development, Porto de Galinhas, Brazil, March 21-25, 2011
[12] M. Inostroza, E. Tanter, E. Bodden, "Join Point Interfaces for Modular Reasoning in Aspect-Oriented Programs", 19th ACM SIGSOFT Symposium and the 13th European Conference on Foundations of Software Engineering, Szeged, Hungary, September 5-9, 2011

[13] J. Woodcock, J. Davies, Using Z: Specification, Refinement, and Proof, Prentice-Hall, 1996

[14] G. Smith, The Object-Z Specification Language, Kluwer Academic Publishers, 2000

[15] C. V. Silva, R. Villarroel, "JPI UML: UML Class and Sequence Diagrams Proposal for Aspect-Oriented JPI Applications", 33rd International Conference of the Chilean Computer Science Society, Talca, Chile, November 8-14, 2014

[16] S. Apel, D. Batory, C. Kastner, G. Saake, Feature-Oriented Software Product Lines: Concepts and Implementation, Springer, 2016 